\title{
Periodic solutions of second-order differential equations with multiple delays
}

\author{
Haibo Chen ${ }^{1 *}$, Hongwu Tang ${ }^{1,2}$ and Juntao Sun ${ }^{3}$
}

* Correspondence:

math_chb@mail.csu.edu.cn

${ }^{1}$ School of Mathematical Science

and Computing Technology,

Central South University, Changsha

410075, P. R. China

Full list of author information is

available at the end of the article

\section{Abstract}

By using the critical point theory and $S^{1}$ index theory, we obtain a new result for the existence and multiplicity of periodic solutions for a class of second-order delay differential equations $x^{\prime \prime}(t)=f(x(t))-[f(x(t-1))+f(x(t-2))+\ldots+f(x(t-(N-1)))]$.

Keywords: delay differential equations, multiple periodic so1utiois, critical point theory, index theory

\section{Introduction}

Inspired by the excellent study in [1], many authors [2-16] studied the following differential delay equations

$$
x^{\prime}(t)=-[f(x(t-1))+f(x(t-2))+\cdots+f(x(t-(N-1)))],
$$

where $f \in C(\mathbb{R}, \mathbb{R})$, and $N \geq 2$ is an integer.

Kaplan and Yorke [2] introduced a technique of couple system which allows them to reduce the search for periodic solutions of a differential delay equation to the problem of finding periodic solutions for a related system of ordinary differential equations. They study periodic solutions of (1.1) with $N=2, f \in C(\mathbb{R}, \mathbb{R})$ is odd, $x f(x)>0$ for $x \neq$ 0 and $f$ satisfies some suitable conditions near 0 and $\infty$. More precisely, if the solution $x(t)$ of (1.1) with $N=2$ satisfies $x(t)=-x(t-2)$, let

$$
x_{1}(t)=x(t), x_{2}(t)=x(t-1),
$$

then $X(t)=\left(x_{1}(t), x_{2}(t)\right)^{T}$ satisfies

$$
X^{\prime}(t)=A_{2} \nabla H(X),
$$

where, $A_{2}=\left(\begin{array}{cc}0 & -1 \\ 1 & 0\end{array}\right)$,

i.e., $A_{2}$ is a skew symmetric matrix, and

$$
H(X)=\int_{0}^{x_{1}} f(s) d s+\int_{0}^{x_{2}} f(s) d s .
$$

$\nabla H(X)$ is the gradient of $H$.

In fact, by direct computation, one has the following proposition:

(c) 2012 Chen et al. ; licensee Springer. This is an open access article distributed under the terms of the Creative Commons Attribution License (http://creativecommons.org/licenses/by/2.0), which permits unrestricted use, distribution, and reproduction in any medium, provided the original work is properly cited. 
Proposition 1.1. (i) Any solution $x(t)$ of (1.1) with $N=2$, and $x(t)=-x(t-2)$ will give a solution of (1.3) $X(t)=\left(x_{1}(t), x_{2}(t)\right)^{T}$ by (1.2). Moreover, $X(t)$ has the following symmetric structure

$$
x_{1}(t)=-x_{2}(t-1), x_{2}(t)=x_{1}(t-1),
$$

(ii) Any solution $X(t)=\left(x_{1}(t), x_{2}(t)\right)^{T}$ of (1.3) with the symmetric structure (1.5) will give a solution of (1.1) by letting $x(t)=x_{1}(t)$. Moreover, $x(t+2)=-x(t)$.

Kaplan and Yorke proved that (1.3) has periodic solutions with the symmetric structure (1.5), which give the Kaplan-Yorke type periodic solutions of (1.1) with period 4, i. e., $x(t)$ satisfying $x(t)=-x(t-2)$. They further conjectured that similar result should be true for the general case $N \geq 2$, i.e., under similar conditions for $f$, (1.1) has a $2 N$-periodic Kaplan-Yorke type periodic solution $x(t)$, i.e., $x(t)$ satisfying $x(t)=-x(t-N)$.

$\mathrm{Li}$ and $\mathrm{He}$ [6-8], in an attempt to reuse Kaplan and Yorke's original idea, applied Lyapunov Center Theorem and some known results about convex Hamiltonian systems [17, Theorem 7.2] to obtain 4-periodic solutions of (1.4). But those 4-periodic solutions obtained by [17, Theorem 7.2] give no information about the symmetric structure (1.5) or the minimal period. The solutions of (1.3), which will not generate noncontact solutions of (1.1), see [14, Remark 3.3].

Herz [12] study (1.1) with $N=2$ by Lyapunov direct method. And, Jekel and Johnston [13], proved the existence of a $2 \mathrm{~N}$-periodic Kaplan-Yorke type periodic solution for (1.1) by Kaplan-Yorke original method and homotopic method.

Fei $[14,15]$ applied the pseudo-index theory [17-20] to obtain periodic solution in a subspace, which surely have the required symmetric structure (1.5) and give solutions to $(1.1)$.

In recent years, Guo and $\mathrm{Yu}$ [16] considered (1.1) with $N=2$ by variational methods directly, and they obtain the Kaplan-Yorke type periodic solutions. That is to say that they do not necessarily transform the existence problem of (1.1) to existence problems for related systems (1.3). Afterwards, Cheng and $\mathrm{Hu}$ [21] studied (1.1) with $N=2$ by Guo-Yu's method in [16]. Guo [22] studied the following second-order differential delay equation by Guo-Yu's method in [16]

$$
x^{\prime \prime}(t)=-f(x(t-r)),
$$

they obtained the multiplicity results for periodic solutions, but the solutions are not Kaplan- Yorke type.

The authors [23,24] considered the Kaplan-Yorke type periodic solutions of (1.1) with $N=2$ by Maslov-type index [25] and Morse theory [26], respectively.

Recently, some researchers [27-31] have begun to study the existence of solutions for second-order differential delay equation by using a variational method. However, to the best of authors' knowledge, the study of Kaplan-Yorke type periodic solutions of second- order differential delay equation using a variational method has received considerably less attention. We find the method apply in [6-15], such as the structure of variational does not directly apply to second-order differential delay equation.

Motivated by the study in [7-16,21-24,27-32], in this article we are concerned with the existence of Kaplan-Yorke type periodic solutions of the following second-order 
differential delay equations

$$
x^{\prime \prime}(t)=f(x(t))-[f(x(t-1))+f(x(t-2))+\cdots+f(x(t-(N-1)))],
$$

where $f \in C(\mathbb{R}, \mathbb{R})$ is odd, and $N \geq 2$ is an integer

If $x(t)=x(t+N)$, let

$$
x_{1}(t)=x(t), x_{2}(t)=x(t-1), \ldots, x_{N}(t)=x(t-(N-1)),
$$

then $z(t)=\left(x_{1}(t), x_{2}(t), \ldots, x_{N}(t)\right)^{T}$ satisfies

$$
z^{\prime \prime}(t)=A_{N} \nabla H(z)
$$

where $A_{N}=\left(\begin{array}{cccc}1 & -1 & \cdots & -1 \\ -1 & 1 & \ddots & \vdots \\ \vdots & \ddots & \ddots & -1 \\ -1 & \cdots & -1 & 1\end{array}\right)$

i.e., $A_{N}$ is a $n \times n$ symmetric matrix, and

$$
H(z)=\int_{0}^{x_{1}} f(s) d s+\int_{0}^{x_{2}} f(s) d s+\cdots+\int_{0}^{x_{N}} f(s) d s .
$$

In fact, by direct computation, one has the following proposition.

Proposition 1.2. (i) Any solution $x(t)$ of (1.7) with $x(t)=x(t-N)$ will give a solution of $(1.9) X(t)=\left(x_{1}(t), x_{2}(t), \ldots, x_{N}(t)\right)^{T}$ by (1.8). Moreover, $X(t)$ has the following symmetric structure

$$
\begin{aligned}
& x_{1}(t)=x_{N}(t-1), x_{2}(t)=x_{1}(t-1), \\
& x_{3}(t)=x_{2}(t-1), \ldots, x_{N}(t)=x_{N-1}(t-1) .
\end{aligned}
$$

(ii) Any solution $X(t)=\left(x_{1}(t), x_{2}(t), \ldots, x_{N}(t)\right)^{T}$ of (1.9) with the symmetric structure (1.11) will give a solution of (1.7) by letting $x(t)=x_{1}(t)$. Moreover, $x(t+N)=x$ $(t)$.

Throughout this article, we always assume that:

(f1) $f \in C(\mathbb{R}, \mathbb{R})$ is odd and $0<\alpha, \beta<+\infty$

$$
\lim _{x \rightarrow 0} \frac{f(x)}{x}=\alpha, \quad \lim _{x \rightarrow \infty} \frac{f(x)}{x}=\beta ;
$$

$\left(f 2^{ \pm}\right)|f(x)-\beta x|$ is bounded and $G_{\beta}(x) \rightarrow \pm \infty$, as $|x| \rightarrow \infty$; $\left(f 3^{ \pm}\right) \pm G \alpha(x)>0$ for $|x|>0$ being small, where $F(x)=\int_{0}^{x} f(s) d s$, and

$$
G_{\beta}(x)=F(x)-\frac{1}{2} \beta x^{2}, G_{\alpha}(x)=F(x)-\frac{1}{2} \alpha x^{2} .
$$

Similarly to the argument in [14,20,33], for given a number $\alpha \in \mathbb{R}$, and $N \geq 2, k \geq 1$ being two integer, we set: 


$$
i(\alpha, N)=\left\{\text { numbers of elements of }\left\{k \mid\left(\frac{2 \pi}{N}(2 k-1)\right)^{2}-2 \alpha<0,(2 k-1 \bmod N) \neq 0 .\right\}\right\}
$$

and

$$
v(\alpha, N)=\left\{\text { numbers of elements of }\left\{k \mid\left(\frac{2 \pi}{N}(2 k-1)\right)^{2}-2 \alpha=0,(2 k-1 \bmod N) \neq 0 .\right\}\right\} .
$$

For convenience, denote \#(1.7) = the number of geometrically different nonconstant periodic solutions of (1.7) which satisfy $x(t-N / 2)=-x(t), \forall t \in R$.

Our main result reads as:

Theorem 1.1. Suppose $f$ satisfies $(f 1)$ and $N \geq 2$ being an integer in (1.7). We have the following conclusions:

(i) \#(1.7) $\geq i(\alpha, N)-i(\beta, N)$ provided $v(\beta, N)=0$ or $(f 2-)$ holds.

(ii) $\#(1.7) \geq i(\alpha, N)-i(\beta, N)+v(\alpha, N)$ provided $(f 3+)$ holds and either $v(\beta, N)=0$ or

$\left(f 2^{-}\right)$holds.

(iii) \#(1.7) $\geq i(\beta, N)+v(\beta, N)-i(\alpha, N)$ provided (f 3-) and $(f 2+)$ holds.

\section{Variational structure}

For $S^{1}=\mathbb{R} /(N \mathbb{Z})$,let $E=H^{1}\left(S^{1}, \mathbb{R}^{N}\right)$. Then $E$ is a Hilbert space with norm $\|\cdot\|$ and inner product $\langle$,$\rangle , and E$ consists of those $z(t)$ in $L^{2}\left(S^{1}, \mathbb{R}^{N}\right)$ whose Fourier series

$$
z(t)=a_{0}+\sum_{m=1}^{\infty}\left(a_{m} \cos \left(\frac{2 \pi}{N} m t\right)+b_{m} \sin \left(\frac{2 \pi}{N} m t\right)\right),
$$

satisfies

$$
\|z\|^{2}=N\left|a_{0}\right|^{2}+\frac{N}{2} \sum_{m=1}^{\infty}\left(1+\beta_{m}^{2}\right)\left(\left|a_{m}\right|^{2}+\left|b_{m}\right|^{2}\right)<\infty,
$$

where $a_{m}, b_{m} \in \mathbb{R}^{N}$ and $\beta_{m}=\frac{2 \pi m}{N}$.

We can define an operator

$$
\left\langle L_{0} z, y\right\rangle=\int_{0}^{N}\left(A_{N}^{-1} \dot{z}, \dot{y}\right) d t
$$

on $E$. By direct computation, $L_{0}$ is a bounded self-adjoint linear operator on $E$ and

$$
L_{0} z(t)=\sum_{m=1}^{\infty} \frac{\beta_{m}^{2}}{1+\beta_{m}^{2}} A_{N}^{-1}\left(a_{m} \cos \beta_{m} t+b_{m} \sin \beta_{m} t\right) .
$$

By (f1), one can show that $H(z) \in C^{1}\left(\mathbb{R}^{\mathbb{N}}, \mathbb{R}\right)$ and satisfies

$$
|H(z)| \leq d_{1}|z|^{2}+d_{2}, \quad \forall z \in \mathbb{R}^{\mathbb{N}},
$$

where $d_{1}, d_{2}>0$. By using similar arguments as in [14,21], we know that

$$
\varphi(z)=\frac{1}{2}\left\langle L_{0} z, z\right\rangle-\int_{0}^{N} H(z) d t \in C^{1}(E, \mathbb{R})
$$

and critical points of $\phi$ in $E$ are classic solutions of (1.9). 
Let $T_{N}$ be the $N \times N$ matrix given by

$$
T_{N}=\left(\begin{array}{ccccc}
0 & 0 & \cdots & 0 & 1 \\
1 & 0 & \cdots & 0 & 0 \\
0 & 1 & \ddots & \vdots & \vdots \\
\vdots & \ddots & \ddots & 0 & 0 \\
0 & \cdots & 0 & 1 & 0
\end{array}\right)
$$

For $z(t) \in E$, define

$$
\delta z(t)=T_{N} z(t-1) .
$$

Then we have $\delta^{N} z(t)=z(t-N)$, and $G=\left\{\delta, \delta^{2}, \ldots, \delta^{N}\right\}$ is a compact group action over $E$. Moreover, if $\delta z(t)=z(t)$ holds, $z(t)$ has the symmetric structure (1.11).

Lemma 2.1. DenoteSE $=\left\{z \in E: \delta z(t)=z(t), z\left(t-\frac{N}{2}\right)=-z(t)\right\}$ Then we have

$$
\begin{aligned}
& S E=\left\{z(t)=\sum_{m=1}^{\infty}\left(a_{m} \cos \left(\frac{2 \pi}{N}(2 m-1) t\right)+b_{m} \sin \left(\frac{2 \pi}{N}(2 m-1) t\right)\right):\right. \\
& \left.\left(\begin{array}{l}
a_{m} \\
b_{m}
\end{array}\right) \in \operatorname{span}\left\{\left(\begin{array}{c}
u_{m} \\
w_{m}
\end{array}\right),\left(\begin{array}{c}
-w_{m} \\
u_{m}
\end{array}\right)\right\}\right\},
\end{aligned}
$$

where $\theta_{m}=\frac{2 \pi}{N}(2 m-1)$ and

$$
\begin{aligned}
& u_{m}=\left(1, \cos \theta_{m}, \ldots, \cos (N-1) \theta_{m}\right)^{T}, \\
& w_{m}=\left(0, \sin \theta_{m}, \ldots, \sin (N-1) \theta_{m}\right)^{T} .
\end{aligned}
$$

Proof. For any, $z(t)=a_{0}+\sum_{m=1}^{\infty}\left(a_{m} \cos \left(\frac{2 \pi}{N} m t\right)+b_{m} \sin \left(\frac{2 \pi}{N} m t\right)\right) \in S E$, we must have $z$ $(t-N / 2)=-z(t)$, which implies that

$$
a_{0}=-a_{0}, a_{m}=(-1)^{m+1} a_{m}, b_{m}=(-1)^{m+1} b_{m}, \text { i.e., } a_{m}=b_{m}=0
$$

for even $m$.

this means that for any $z \in S E$,

$$
z(t)=\sum_{m=1}^{\infty}\left(a_{m} \cos \left(\frac{2 \pi}{N}(2 m-1) t\right)+b_{m} \sin \left(\frac{2 \pi}{N}(2 m-1) t\right)\right) .
$$

Note that $\delta z(t)=z(t) \Leftrightarrow T_{N} z(t-1)=z(t)$, i.e.,

$$
\begin{aligned}
& \sum_{m=1}^{\infty}\left(a_{m} \cos \left(\frac{2 \pi}{N}(2 m-1) t\right)+b_{m} \sin \left(\frac{2 \pi}{N}(2 m-1) t\right)\right) \\
& =\sum_{m=1}^{\infty}\left(T_{N} a_{m} \cos \left(\frac{2 \pi}{N}(2 m-1)(t-1)\right)+T_{N} b_{m} \sin \left(\frac{2 \pi}{N}(2 m-1)(t-1)\right)\right)
\end{aligned}
$$

This implies that for $k \geq 1$

$$
\left\{\begin{array}{l}
a_{m}=T_{N} a_{m} \cos \theta_{m}-T_{N} b_{m} \sin \theta_{m} \\
b_{m}=T_{N} a_{m} \sin \theta_{m}+T_{N} b_{m} \cos \theta_{m}
\end{array}\right.
$$


If we introduce complex vector $C_{m}=a_{m}+i b_{m}$, the above (2.7) becomes

$$
C_{m}=e^{i \theta_{m}} T_{N} C_{m} .
$$

Note that

$$
\operatorname{det}\left(T_{N}-\lambda I_{N}\right)=\lambda^{N}-1,
$$

the eigenvalues of $T_{N}$ are

$$
\lambda=e^{-i \frac{2 j \pi}{N}}, j=1,2,3, \ldots,
$$

Equation (2.7) implies that $C_{m}$ must be the eigenvector associated with the eigenvalue

$$
\lambda=e^{-i \theta_{m}},
$$

We obtain that

$$
\begin{aligned}
& C_{m}=\left(1, e^{i \theta_{m}}, \ldots, e^{i(N-1) \theta_{m}}\right)^{T}, \\
& \left\{\begin{array}{l}
u_{m}=\operatorname{Re}\left(C_{m}\right)=\left(1, \cos \theta_{m}, \cos 2 \theta_{m}, \ldots, \cos (N-1) \theta_{m}\right)^{T}, \\
w_{m}=\operatorname{Im}\left(C_{m}\right)=\left(0, \sin \theta_{m}, \sin 2 \theta_{m}, \ldots, \sin (N-1) \theta_{m}\right)^{T} .
\end{array}\right.
\end{aligned}
$$

By direct computation, $i C_{m}$ is also the eigenvector associated with the eigenvalue $\lambda=e^{-i \theta_{m}}$.

Therefore, we have the conclusion. The proof is complete.

Lemma 2.2. Let $\phi$ be given in (2.3) and $\left.\phi\right|_{S E}$ be the restriction of $\phi$ on SE. Then critical points of $\left.\phi\right|_{S E}$ over $S E$ are critical points of $\phi$ over $E$.

Proof. By (1.9) and direct computation, we have

$$
A_{N} T_{N}=T_{N} A_{N}, H\left(T_{N} z\right)=H(z), \nabla H\left(T_{N} z\right)=T_{N} \nabla H(z) .
$$

Combining these with (2.3) and the fact that any $z(t) \in E$ is $N$-periodic, one can easily verify that

$$
\varphi(\delta z)=\varphi(z), \quad \varphi^{\prime}(\delta z)=\delta \varphi^{\prime}(z),
$$

i.e., $\phi$ is $G$-invariant, and $\phi$ ', is $G$-equivariant. The conclusion follows directly.

For any $\alpha \in \mathbb{R}$, define an operator $L_{\alpha}$ by extending the bilinear form

$$
\left\langle L_{\alpha} z, y\right\rangle=\left\langle L_{0} z, y\right\rangle-\int_{0}^{N}(\alpha z, \gamma) d t,
$$

on $E$. Moreover, $L_{\alpha}$ is $G$-equivariant. By direct computation, $L_{\alpha}$ is a bounded selfadjoint linear operator on $E$ and if $z(t) \in S E$

$$
L_{\alpha} z(t)=\sum_{m=1}^{\infty} \frac{1}{1+\beta_{m}^{2}}\left(\beta_{m}^{2} A_{N}^{-1}-\alpha I\right)\left(a_{m} \cos \beta_{m} t+b_{m} \sin \beta_{m} t\right) .
$$

For $m \geq 1$, denote

$$
\operatorname{SE}(m)=\left\{z(t)=a_{m} \cos \left(\frac{2 \pi}{N}(2 m-1) t\right)+b_{m} \sin \left(\frac{2 \pi}{N}(2 m-1) t\right):\left(\begin{array}{l}
a_{m} \\
b_{m}
\end{array}\right) \text { satisfies }(2.5)\right\} .
$$


Then $S E=\oplus_{j=1}^{\infty} S E(j)$. Since $A_{N}=I_{N}-\left(T_{N}+T_{N}^{2}+\cdots+T_{N}^{N-1}\right)$, and $A_{N}^{T}=A_{N}$, so $S E(m)$ is the eigen-subspace of $L_{0}$ corresponding to eigenvalue $\lambda_{m}$, here $\lambda_{m}=2-N$, as (2m $1 \bmod N)=0 ; \lambda_{m}=2$, as $(2 m-1 \bmod N) \neq 0$. Denote by $M-(\cdot), M+(\cdot)$ and $M^{0}(\cdot)$ the positive definite, negative definite and null subspaces of the self-adjoint linear operator defining it, respectively.

Lemma 2.3. For $k \geq 1, \gamma_{k}^{\alpha}=\left(\frac{2 \pi}{N}(2 k-1)\right)^{2} \lambda_{k}^{-1}-\alpha$. Then $L_{\alpha}$, as an operator on $S E$, has the following properties on SE.

$$
\begin{aligned}
& M^{-}\left(L_{\alpha}\right)=\underset{k=1, \gamma_{k}^{\alpha}<0}{\infty} S E(k), \\
& M^{+}\left(L_{\alpha}\right)=\underset{k=1, \gamma_{k}^{\alpha}>0}{\infty} S E(k), \\
& M^{0}\left(L_{\alpha}\right)=\bigoplus_{k=1, \gamma_{k}^{\alpha}=0}^{\infty} S E(k) .
\end{aligned}
$$

Proof. For $m \geq 1$ and $z_{m}=a_{m} \cos \left(\frac{2 \pi}{N}(2 m-1) t\right)+b_{m} \sin \left(\frac{2 \pi}{N}(2 m-1) t\right)$, consider the eigenvalue problem

$$
L_{\alpha} z_{m}=\lambda_{m}^{\alpha} z_{m}
$$

By (2.7) and (2.11), we have

$$
\begin{aligned}
& \frac{1}{1+\theta_{m}^{2}}\left(\theta_{m}^{2} A_{N}^{-1}-\alpha I\right) a_{m}=\lambda_{m}^{\alpha} a_{m} \\
& \frac{1}{1+\theta_{m}^{2}}\left(\theta_{m}^{2} A_{N}^{-1}-\alpha I\right) b_{m}=\lambda_{m}^{\alpha} b_{m} .
\end{aligned}
$$

Since $a_{m}, b_{m}$ are the eigenvector of $A_{N}$ corresponding to eigenvalue $\lambda_{m}$, then $\lambda_{m}^{\alpha}=\left(1+\theta_{m}^{2}\right)^{-1}\left(\theta_{m}^{2} \lambda_{m}^{-1}-\alpha\right)$. Therefore $L_{\alpha}$ is positive definite, negative definite, or null on $S E(k)$ if and only if $\gamma_{k}^{\alpha}=\left(\frac{2 \pi}{N}(2 k-1)\right)^{2} \lambda_{k}^{-1}-\alpha$ is positive, negative, or zero, respectively.

This implies (2.12) directly.

For $S^{1}=\mathbb{R} /(N \mathbb{Z})$, there is a natural $S^{1}$-action over $S E$, defined by

$$
T(\theta) z(t)=z(t+\theta), \forall \theta \in S^{1}, \forall z \in S E .
$$

It is easy to see that $\phi$ is $S^{1}$-invariant, $\phi^{\prime}$ is $S^{1}$-equivariant, and

$$
\operatorname{Fix}\left(S^{1}\right)=\left\{u \in S E: T(\theta) u=u, \forall \theta \in S^{1}\right\}=\{0\} .
$$

By directly applying [20, Theorem 2.4] to $\phi$ over $S E$, we have the following lemma.

Lemma 2.4. Assume there exist two closed $S^{1}$-invariant linear subspaces, $S E^{+}$and $S E^{-}$ , of $S E$ and $r>0$ such that (a) $\left(S E++S E^{-}\right)$is closed and of finite codimension in $S E$,

(b) $L\left(S E^{-}\right) L S E^{-}$with $L=L_{\alpha}$ or $L=L_{\beta}$,

(c) there exist $c_{0} \in \mathbb{R}, c_{0}>-\infty$ such that

$$
\inf _{z \in S E^{+}} \varphi(z)=c_{0}
$$

(d) there exists $c_{\infty} \in \mathbb{R}$ such that

$$
\varphi(z) \leq c_{\infty}<\varphi(0), \forall z \in\left(S E^{-} \cap S_{r}\right)=\left\{z \in S E^{-}: z=r\right\},
$$

(e) $\phi$ satisfies $(P S) c$ condition for $c_{0} \leq c \leq c_{\infty}$, i.e., every sequence $\left\{z_{m}\right\} \subseteq S E$ with $\phi\left(z_{m}\right) \rightarrow$ $c$ and $\phi^{\prime}\left(z_{m}\right) \rightarrow 0$ possesses a convergent subsequence. Then $\phi$ possesses at 
least $\frac{1}{2}\left[\operatorname{dim}\left(S E^{-} \cap S E^{+}\right)-\operatorname{codim}_{S E}\left(S E^{-}+S E^{+}\right)\right]$geometrically different critical orbits in $\phi^{-1}\left(\left[c_{0}, c_{\infty}\right]\right)$.

\section{Proof of main results}

Proof of Theorem 1.1 As we already proved in Lemma 2.2, critical points of $\phi$ over $S E$ are critical points of $\phi$ over $E$. Hence they are nonconstant classic $N$-periodic solutions of (1.7) with the symmetric structure (1.11). By Proposition 1.2, they give solutions of (1.7) with the property $x(t-N / 2)=-x(t)$. Therefore, we can seek critical points of $\phi$ on $S E$ directly.

Set:

$$
\psi_{\beta}(z)=\int_{0}^{N}\left[H(z)-\left(\frac{1}{2} \beta z, z\right)\right] d t, \quad \psi_{\alpha}(z)=\int_{0}^{N}\left[H(z)-\left(\frac{1}{2} \alpha z, z\right)\right] d t
$$

Then

$$
\varphi(z)=\frac{1}{2}\left\langle L_{\alpha} z, z\right\rangle-\psi_{\alpha}(z), \quad \varphi(z)=\frac{1}{2}\left\langle L_{\beta} z, z\right\rangle-\psi_{\beta}(z)
$$

Case (i): If $i(\alpha, N)>i(\beta, N)$. We shall carry out the proof in several steps.

Step 1: let

$$
\psi_{\alpha}(0)=0, \quad \frac{\left\|\psi_{\alpha}^{\prime}(z)\right\|}{\|z\|} \rightarrow 0, \text { as }\|z\| \rightarrow 0,
$$

Then Lemma 2.4(a)and (b) hold with $L=L_{\alpha}$. By (f1), using the same argument as [18, Lemma 5.5], it is easy to show that

$$
\psi_{\alpha}(0)=0, \quad \frac{\left\|\psi_{\alpha}^{\prime}(z)\right\|}{\|z\|} \rightarrow 0, \text { as }\|z\| \rightarrow 0,
$$

We denote

$$
\lambda^{-}=\min \left\{\left|\gamma_{m}^{\alpha}\right|\left(1+\theta_{m}^{2}\right)^{-1} \mid \gamma_{m}^{\alpha}<0, m \in \mathbb{N}\right\},
$$

and

$$
\lambda^{+}=\min \left\{\left(1+\theta_{m}^{2}\right)^{-1} \gamma_{m}^{\beta} \mid \gamma_{m}^{\beta}>0, m \in \mathbb{N}\right\} .
$$

Since $\psi_{\alpha}(0)=0, \frac{\left\|\psi_{\alpha}^{\prime}(z)\right\|}{\|z\|} \rightarrow 0$, as $\|z\| \rightarrow 0$, for $\varepsilon=\frac{\lambda^{-}}{4}$, there exists a constant $r>0$ such that for any $z \in H^{1}\left(S^{1}, \mathbb{R}^{N}\right)$

$$
\left\|\psi_{\alpha}^{\prime}(z)\right\| \leq \frac{\lambda^{-}}{4}\|z\|, \quad \text { as }\|z\| \leq r .
$$

Furthermore,

$$
\begin{aligned}
\left|\psi_{\alpha}(z)\right| & =\left|\psi_{\alpha}(z)-\psi_{\alpha}(0)\right| \\
& =\left|\int_{0}^{1} \frac{\partial \psi_{\alpha}(\tau z)}{\partial \tau} d \tau\right| \\
& \leq \int_{0}^{1}\left|\left\langle\psi_{\alpha}^{\prime}(\tau z), z\right\rangle\right| d \tau \\
& \leq \frac{\lambda^{-}}{4}\|z\|^{2} .
\end{aligned}
$$


Therefore, we have for $z \in\left(E^{-} \cap S_{r}\right)=\left\{z \in E^{-} \mid\|z\|=r\right\}$

$$
\begin{aligned}
\varphi(z) & =\frac{1}{2}\left\langle L_{\alpha} z, z\right\rangle-\psi_{\alpha}(z) \\
& \leq \frac{-\lambda^{-}}{2}\|z\|^{2}+\frac{\lambda^{-}}{4}\|z\|^{2} \\
& =\frac{-\lambda^{-}}{4}\|z\|^{2} \\
& =\frac{-\lambda^{-}}{4} r^{2}<0 .
\end{aligned}
$$

Thus (d) of Lemma 2.4 holds.

If $M^{0}(L \beta)=\{0\}$, by (f1), using the same argument as [18, Lemma 5.5], it is easy to show that

$$
\frac{\left\|\psi_{\alpha}^{\prime}(z)\right\|}{\|z\|} \rightarrow 0, \text { as }\|z\| \rightarrow \infty,
$$

it follows that given $\varepsilon=\frac{\lambda^{+}}{2}>0$, there exists $r>0$, such that

$$
\left\|\psi_{\alpha}^{\prime}(z)\right\| \leq \frac{\lambda^{+}}{2}\|z\|,\|z\|>r .
$$

Moreover, there is $d_{1}>0$ such that

$$
\left\|\psi_{\alpha}^{\prime}(z)\right\| \leq d_{1},\|z\| \leq r
$$

thus

$$
\left\|\psi_{\alpha}^{\prime}(z)\right\| \leq \frac{\lambda^{+}}{2}\|z\|+d_{1}, \forall z \in H^{1}\left(S^{1}, \mathbb{R}^{N}\right) .
$$

Using the above formula we get

$$
|\nabla H(z)-\beta z| \leq \frac{\lambda^{+}}{2}|z|+d_{1} .
$$

Since for any $z \in S E^{+}=M^{+}(L \beta) \oplus M^{0}(L \beta)$

$$
\begin{aligned}
\left|\psi_{\beta}(z)\right| & =\left|\psi_{\beta}(z)-\psi_{\beta}(0)\right| \\
& =\left|\int_{0}^{1} \frac{\partial \psi_{\beta}(\tau z)}{\partial \tau} d \tau\right| \\
& =\left|\int_{0}^{1}\left\langle\psi_{\beta}^{\prime}(\tau z), z\right\rangle d \tau\right| \\
& \leq \int_{0}^{1} \frac{\lambda^{+} \tau}{2} z^{2}+d_{1} \cdot z d \tau \\
& \leq \frac{\lambda^{+}}{4} z^{2}+d_{1} z .
\end{aligned}
$$


Then for any $z \in S E^{+}=M^{+}(L \beta) \oplus M^{0}(L \beta)=M^{+}(L \beta) \oplus\{0\}$

$$
\begin{aligned}
\varphi(z) & =\frac{1}{2}\left\langle L_{\beta} z, z\right\rangle-\psi_{\beta}(z) \\
& \geq \frac{\lambda^{+}}{2} z^{2}-\frac{\lambda^{+}}{4} z^{2}-d_{1} z \\
& =\frac{\lambda^{+}}{4} z^{2}-d_{1} z .
\end{aligned}
$$

Thus there exists $c_{0}>-\infty$ such that (c) of Lemma 2.4 holds.

If $M^{0}\left(L_{\beta}\right) \neq\{0\}$, by $\left(f 2^{-}\right)$and (1.12), for any $M>0$, there exists a constant $d_{2}>0$ such that

$$
G_{\beta}(z) \leq-d_{2}, \quad|z|>M
$$

Let

$$
\Omega_{1}=\{t \in[0, N]|| z \mid>M\}
$$

and

$$
\Omega_{2}=\{t \in[0, N]|| z \mid \leq M\} .
$$

Since $F(z) \in C^{2}\left(\mathbb{R}^{N}, \mathbb{R}\right)$, by (3.3), there exists a constant $d_{3}>0$ such that

$$
\int_{\Omega_{2}}\left[G_{\beta}(z(t)), z(t)\right] d t \leq d_{3}, \quad|z| \leq M .
$$

By (3.1) - (3.4), we have

$$
\psi_{\beta}(z)=\int_{0}^{N}\left[H(z)-\left(\frac{1}{2} \beta z, z\right)\right] d t \leq d_{3}
$$

Then we have for $z=z^{+}+z^{0} \in M^{+}\left(L_{\beta}\right) \oplus M^{0}\left(L_{\beta}\right)$

$$
\begin{aligned}
\varphi(z) & =\frac{1}{2}\left\langle L_{\beta} z, z\right\rangle-\psi_{\beta}(z) \\
& =\frac{1}{2}\left\langle L_{\beta} z^{+}, z^{+}\right\rangle-\psi_{\beta}(z) \\
& \geq-d_{3} .
\end{aligned}
$$

Thus $\phi$ is bounded from below on $E^{+}$. Therefore, condition (c) of Lemma 2.4 is verified.

Step 2: Using the same argument as [22,25] and [26, Lemma 4.2], one can prove that $\phi$ satisfies $(P S) c$ condition for any $c \in \mathbb{R}$ under the condition either $(f 1)$ with $v(\beta, N)=$ 0 holds or $\left(f 2^{ \pm}\right)$holds.

Step 3: By Lemma (2.4), $\phi$ has at least $\sigma=\frac{1}{2}\left[\operatorname{dim}\left(S E^{-} \cap S E^{+}\right)-\operatorname{codim}_{S E}\left(S E^{-}+S E^{+}\right)\right]$ geometrically different critical orbits in $\phi^{-1}\left(\left[c_{0}, c_{\infty}\right]\right)$.

Now by Lemma (2.3), it is easy to show that

$$
\begin{aligned}
\sigma & =\frac{1}{2}\left[\operatorname{dim}\left(S E^{-} \cap S E^{+}\right)-\operatorname{codim}_{S E}\left(S E^{-}+S E^{+}\right)\right] \\
& =\frac{1}{2}\left[\operatorname{dim} M^{-}\left(L_{\alpha}\right)-\operatorname{dim}^{-}\left(L_{\beta}\right)\right] \\
& =\frac{1}{2}[2 i(\alpha, N)-2 i(\beta, N)] .
\end{aligned}
$$


This means \#(1.7) $\geq i(\alpha, N)-i(\beta, N)$, and this completes the proof of case (i).

For case (ii), (iii), using the same idea and similar arguments, one can show that the conclusions hold. We omit the details. The proof is complete.

Example. Consider the following equation

$$
x^{\prime \prime}(t)=f(x(t))-f(x(t-1)),
$$

and its coupled system

$$
x^{\prime \prime}(t)=f(x)-f(\gamma), \gamma^{\prime \prime}(t)=-f(x)+f(y) .
$$

with $f \in C$ ( $\mathbb{R}, \mathbb{R}$ ) being odd and

$$
\begin{aligned}
& f(x)=\alpha x+\alpha_{0} x^{\frac{1}{3}}, \text { for }|x| \geq 100 \\
& f(x)=\beta x+\beta_{0} x^{3}, f \circ|x| \leq 1 .
\end{aligned}
$$

Here $N=2$, and by Definition

$$
i(\alpha, 2)=\sharp\left\{k \mid(\pi(2 k-1))^{2}-2 \alpha<0, k=1,2, \ldots\right\},
$$

and

$$
v(\alpha, 2)=\sharp\left\{k \mid(\pi(2 k-1))^{2}-2 \alpha=0, k=1,2, \ldots\right\} .
$$

Let $\alpha=16, \beta=1$. Then it is easy to see that

$$
i(16,2)=2, v(16,2)=0, i(1,2)=0, v(1,2)=0
$$

By Theorem 1.1, Equation (3.5) has at least $i(16,2)-i(1,2)=2$ geometrically different nonconstant 2-periodic solutions which satisfy $x(t-1)=-x(t)$.

\section{Acknowledgements}

The authors thank the referees for valuable comments and suggestions which improved the presentation of this manuscript. This study was partially supported by NFSC (10871206, 10961011, 60964006, 11161013) Guangxi Natural Science Foundation (2011GXNSFA018134).

\section{Author details}

${ }^{1}$ School of Mathematical Science and Computing Technology, Central South University, Changsha 410075, P. R. China ${ }^{2}$ School of Mathematics and Computing Science, Guilin University of Electronic Technology, Guilin 541004, P. R. China

${ }^{3}$ School of Science, Shandong University of Technology, Zibo, Shandong 255049, P. R. China

\section{Authors' contributions}

All authors carried out the proof and authors conceived of the study. All authors read and approved the final manuscript.

\section{Competing interests}

The authors declare that they have no competing interests.

Received: 14 October 2011 Accepted: 11 April 2012 Published: 11 April 2012

\section{References}

1. Grafton, R: A periodicity theorem for autonomous functional differential equations. J Diff Equ. 6, 87-109 (1969). doi:10.1016/0022-0396(69)90119-3

2. Kaplan, JL, Yorke, JA: Ordinary differential equations which yield periodic solution of delay equations. J Math Anal Appl. 48, 317-324 (1974). doi:10.1016/0022-247X(74)90162-0

3. Kaplan, JL, Yorke, JA: On the stability of a periodic solution of a differential delay equation. SIAM J Math Anal. 6 , 268-282 (1975). doi:10.1137/0506028

4. Kaplan, JL, Yorke, JA: On the nonlinear differential delay equation $x^{\prime}(t)=-f(x(t), x(t-1))$. J Diff Equ. 23, 294-314 (1977)

5. Nussbaum, RD: Uniqueness and nonuniqueness for periodic solutions of $x^{\prime}(t)=-g(x(t-1))$. J Diff Equ. 34, 25-54 (1979). doi:10.1016/0022-0396(79)90016-0

6. Li, JB, He, XZ: Multiple periodic solutions of differential delay equations created by asymptotically linear Hamiltonian systems. Nonlinear Anal TMA. 31, 45-54 (1998). doi:10.1016/S0362-546X(96)00058-2 
7. Li, JB, He, XZ: Proof and generalization of Kaplan-Yorke's conjecture on periodic solution of differential delay equations. Sci China (Ser A). 42(9):957-964 (1999). doi:10.1007/BF02880387

8. Li, JB, He, XZ, Liu, ZR: Hamiltonian symmetric groups and multiple periodic solutions of differential delay equations. Nonlinear Anal. 35, 457-474 (1999). doi:10.1016/50362-546X(97)00623-8

9. Ge, WG: Number of simple periodic solutions of differential difference equation on $x^{\prime}(t)=-f(x(t-1))$. Chinese Ann Math. 14A, 480-491 (1993)

10. Chen, YS: The existence of periodic solutions of the equation $x^{\prime}(t)=-f(x(t), x(t-r))$. J Math Anal Appl. 163, 227-237 (1992). doi:10.1016/0022-247X(92)90290-T

11. Chen, YS: The existence of periodic solutions for a class of neutral differential difference equations. Bull Austral Math Soc. 33, 508-516 (1992). doi:10.1017/S0334270000007190

12. Herz, AVM: Solutions of $x^{\prime}(t)=-g(x(t-1))$ approach the Kaplan-Yorke orbits for odd sigmoidg. J Diff Equ. 118, 36-53 (1995). doi:10.1006/jdeq.1995.1066

13. Jekel, S, Johnston, C: A Hamiltonian with periodic orbits having several delays. J Diff Equ. 222, 425-438 (2006). doi:10.1016/j.jde.2005.08.013

14. Fei, GH: Multiple periodic solutions of differential delay equations via Hamiltonian systems (I). Nonlinear Anal. 65, 25-39 (2006). doi:10.1016/j.na.2005.06.011

15. Fei, GH: Multiple periodic solutions of differential delay equations via Hamiltonian systems (II). Nonlinear Anal. 65, 40-58 (2006). doi:10.1016/.na.2005.06.012

16. Guo, ZM, Yu, JS: Multiplicity results for periodic solutions to delay differential difference equations via critical point theory. J Diff Equ. 218, 15-35 (2005). doi:10.1016/j.jde.2005.08.007

17. Mawhin, J, Willem, M: Critical point theory and Hamiltonian systems. Springer, New York (1989)

18. Benci, V: On critical point theory for indefinite functionals in the presence of symmetries. Trans Am Math Soc. 274, 533-572 (1982). doi:10.1090/S0002-9947-1982-0675067-X

19. Chang, KC: Infinite Dimensional Morse Theory and Multiple Solution Problems. Birkhaăser, Bostoi (1993)

20. Fannio, LO: Multiple periodic solution of Hamiltonian systems with strong resonance at infinity. Discrete Cont Dyn Syst. 3, 251-264 (1997)

21. Cheng, $\mathrm{R}, \mathrm{Hu}, \mathrm{JH}$ : Variational Approaches for the Existence of Multiple Periodic Solutions of Differential Delay Equations J Abstr Appl Anal 2010, 14 (2010). Article ID 978137 doi:10.1155/2010/978137

22. Guo, CJ, Guo, ZH: Existence of multiple periodic solutions for a class of second-order delay differential equations. Nonlinear Anal RWA. 10, 3285-3297 (2009). doi:10.1016/j.nonrwa.2008.10.023

23. Cheng, R, Zhang, DF: Existence results of periodic solutions for non-autonomous differential delay equations with asymptotically linear properties. Nonlinear Anal RWA. 11, 3406-3412 (2010). doi:10.1016/j.nonrwa.2009.11.031

24. Zhang, XS, Meng, Q: Nontrivial periodic solutions for delay differential systems via Morse theory. Nonlinear Anal. 74, 1960-1968 (2011). doi:10.1016/.na.2010.11.003

25. Long, YM, Zehnder, E: Morse theory for forced oscillations of asymptotically linear Hamiltonian systems. In: Albeverio $\mathrm{S}$ (ed.) Stochastic processes, Physics and Geometry. pp. 528-563. Proceedings of the Conference in Asconal/Locarno, Switzerland, World Scientific, Singapore (1990)

26. Li, SJ, Liu, JQ: Morse theory and asymptotically linear Hamiltonian systems. J Diff Equ. 78, 53-73 (1989). doi:10.1016/ 0022-0396(89)90075-2

27. Shu, $\mathrm{XB}, \mathrm{Xu}, \mathrm{YT}$ : Multiple periodic solutions to a class of second-order functional differential equations of mixed type. Acta Math Appl Sin. 29(5):821-831 (2006)

28. Wang, GQ, Yan, JR: Existence of periodic solutions for second-order nonlinear neutral delay equations. Acta Math Sin. 47(2):379-384 (2004)

29. Wang, GQ, Cheng, SS: Even periodic solutions of higher order duffing differential equations. J Czechoslovak Math. 57(132):331-343 (2007)

30. Xu, YT, Guo, ZM: Applications of a Zp index theory to periodic solutions for a class of functional differential equations. J Math Anal Appl. 257(1):189-205 (2001). doi:10.1006/jmaa.2000.7342

31. Xu, YT, Guo, ZM: Applications of a geometrical index theory to functional differential equations. Acta Math Sin. 44(6):1027-1036 (2001)

32. Lu, SP, Ge, WG: Periodic solutions of the second-order differential equation with deviating Arguments. Acta Math Sin. 45, 811-818 (2002)

33. Schechter, M: Spectra of Partial Differential Equation. North-Holland, Amsterdam (1971)

doi:10.1186/1687-1847-2012-43

Cite this article as: Chen et al:: Periodic solutions of second-order differential equations with multiple delays.

Advances in Difference Equations 2012 2012:43. 